\title{
Benzodiazepine prescribing patterns and drug overdose mortality among individuals receiving opioid analgesics
}

\author{
Tae Woo Park ${ }^{1,2^{*}}$, Richard Saitz ${ }^{3,4}$, Dara Ganoczy ${ }^{5}$, Mark A llgen ${ }^{5,6}$, Amy SB Bohnert ${ }^{5,6}$ \\ From 2014 Addiction Health Services Research (AHSR) Conference \\ Boston, MA, USA. 15-17 October 2014
}

\section{Background}

Drug overdose mortality, particularly involving opioid analgesics, has risen steadily over the past two decades and is now the leading cause of injury death in the United States. Benzodiazepines are commonly prescribed concurrently to individuals receiving opioid analgesics. Little is known about the association between benzodiazepine-prescribing patterns and overdose mortality in those receiving opioid analgesics. We aimed to study the association between benzodiazepine prescription history, dose, type and dosing schedule, and the risk of drug overdose mortality among individuals receiving opioid analgesics.

\section{Materials and methods}

This was a case-cohort study utilizing Veterans Health Administration (VHA) administrative data. Participants were all individuals who died of a drug overdose $(\mathrm{n}=$ 2326) while receiving opioid analgesics and a random sample of individuals $(\mathrm{n}=404725)$ who received VHA medical services and opioid analgesics between fiscal years 2004 and 2009. Benzodiazepine prescription history, dose, type, and dosing schedule were determined by dispensation data from the VHA's Pharmacy Benefits Management Services. Benzodiazepine prescription history was categorized as periods of time when individuals were currently prescribed, formerly prescribed, or not prescribed benzodiazepines. Drug overdose mortality was based on cause of death information from the National Death Index. Associations between benzodiazepine prescription history, dose, type and dosing schedule, and overdose mortality were examined using Cox proportional hazards models.

\footnotetext{
* Correspondence: tpark1@lifespan.org

'Division of General Internal Medicine, Department of Medicine, Alpert Medical School and Rhode Island Hospital, Providence, RI, 02903, USA
} Full list of author information is available at the end of the article

\section{Results}

Twenty-seven percent of individuals who received opioid analgesics also received benzodiazepines during the study period. Approximately half of the drug overdose deaths $(\mathrm{n}=1162)$ occurred during periods when individuals were concurrently receiving benzodiazepines and opioids. Risk of overdose death increased based on benzodiazepine prescription history: formerly prescribed versus not prescribed, adjusted hazard ratio $(\mathrm{HR})=2.22(95 \%$ confidence interval [CI]; 1.95-2.52; absolute risk difference approximation $[\mathrm{ARDA}]=0.12 \%$ ); currently prescribed versus not prescribed, adjusted $\mathrm{HR}=3.59$ (95\% CI, 3.243.97; ARDA $=0.25 \%$ ). Risk of overdose death increased as daily benzodiazepine dose increased. When compared to clonazepam, temazepam was associated with a decreased overdose risk; adjusted $\mathrm{HR}=0.73$ (95\% CI; 0.56-0.95; ARDA $=0.03 \%$ ). Benzodiazepine dosing schedule was not associated with overdose risk.

\section{Conclusions}

Among individuals receiving opioid analgesics, receipt of benzodiazepines was associated with an increased risk of drug overdose death, and these risks were generally consistent at all levels of opioid dose. The risks and benefits of benzodiazepine-prescribing in this population warrant further evaluation.

\footnotetext{
Authors' details

'Division of General Internal Medicine, Department of Medicine, Alpert Medical School and Rhode Island Hospital, Providence, RI, 02903, USA. ${ }^{2}$ Department of Psychiatry and Human Behavior; Alpert Medical School and Rhode Island Hospital, Providence, RI, 02903, USA. ${ }^{3}$ Clinical Addiction Research and Education (CARE) Unit, Section of General Internal Medicine, Department of Medicine, Boston Medical Center and Boston University School of Medicine, Boston, MA, 02118, USA. ${ }^{4}$ Department of Community Health Sciences, Boston University School of Public Health, Boston, MA,
} 
02118, USA. ${ }^{5}$ Department of Veterans Affairs, Health Services Research \& Development (HSR\&D), Ann Arbor, MI, 48109, USA. ${ }^{6}$ Center of Excellence, and Serious Mental IIIness Treatment Resource and Evaluation Center, Ann Arbor, MI, 48109, USA.

Published: 20 February 2015

doi:10.1186/1940-0640-10-S1-A48

Cite this article as: Park et al:: Benzodiazepine prescribing patterns and drug overdose mortality among individuals receiving opioid analgesics. Addiction Science \& Clinical Practice 2015 10(Suppl 1):A48.

Submit your next manuscript to BioMed Central and take full advantage of:

- Convenient online submission

- Thorough peer review

- No space constraints or color figure charges

- Immediate publication on acceptance

- Inclusion in PubMed, CAS, Scopus and Google Scholar

- Research which is freely available for redistribution

Submit your manuscript at www.biomedcentral.com/submit
() Biomed Central 